\title{
A 4DCT imaging-based breathing lung model with relative hysteresis
}

\author{
Shinjiro Miyawaki, ${ }^{1}$ Sanghun Choi, ${ }^{1}$ Eric A. Hoffman, ${ }^{3,4,5}$ and Ching-Long Lin ${ }^{1,2, *}$ \\ ${ }^{1}$ IIHR-Hydroscience \& Engineering, \\ ${ }^{2}$ Department of Mechanical and Industrial Engineering, \\ ${ }^{3}$ Biomedical Engineering, ${ }^{4}$ Medicine and ${ }^{5}$ Radiology \\ The University of Iowa, Iowa City, Iowa 52242
}

*Corresponding author: Ching-Long Lin

Address: 3131 Seamans Center, Iowa City, Iowa 52242, U.S.A.,

Email: ching-long-lin@uiowa.edu, Tel: +1 319335 5673, Fax: +1 3193355669 


\begin{abstract}
To reproduce realistic airway motion and airflow, the authors developed a deforming lung computational fluid dynamics (CFD) model based on four-dimensional (4D, space and time) dynamic computed tomography (CT) images. A total of 13 time points within controlled tidal volume respiration were used to account for realistic and irregular lung motion in human volunteers. Because of the irregular motion of 4DCT-based airways, we identified an optimal interpolation method for airway surface deformation during respiration, and implemented a computational solid mechanics-based moving mesh algorithm to produce smooth deforming airway mesh. In addition, we developed physiologically realistic airflow boundary conditions for both models based on multiple images and a single image. Furthermore, we examined simplified models based on one or two dynamic or static images. By comparing these simplified models with the model based on 13 dynamic images, we investigated the effects of relative hysteresis of lung structure with respect to lung volume, lung deformation, and imaging methods, i.e., dynamic vs. static scans, on CFD-predicted pressure drop. The effect of imaging method on pressure drop was 24 percentage points due to the differences in airflow distribution and airway geometry.
\end{abstract}

Keywords: multiscale, pulmonary airflow, relative hysteresis, regional ventilation, image registration, $4 D C T$ 


\section{Introduction}

Quantitative computed tomography (CT) has been used to obtain accurate structures of the airways and lobes of the lungs (Hoffman et al. (2006)). An image registration technique can link two static CT images to describe regional lung functions (Yin et al. (2011)), and it has been utilized to characterize functional alterations due to lung diseases (Bhatt et al. (2016), Bodduluri et al. (2013), Choi et al. (2013), Galban et al. (2012)). While two static images can provide linear lung deformation, three static images can reproduce non-linear lung deformation, but in the absence of lung hysteresis (Yin et al. (2013), Jahani et al. (2014)). To better understand regional physiological lung function, we recently acquired four-dimensional (4D, space and time) dynamic CT images along with static images (Jahani et al. (2015)). The study showed the relative hysteresis of anisotropic lung deformation with respect to lung air volume (simply "lung volume” hereafter), and demonstrated that regional ventilation derived from dynamic images is consistent with local air-volume change derived from two static images.

While image registration can provide information on regional ventilation and lung deformation, computational fluid dynamics (CFD) models can provide information regarding gas flow, particle transport, and heat transfer in the human lung (Lin et al. (2007), Miyawaki et al. (2012), Wu et al. (2014)). For example, CFD-based models can predict the location of particle deposition on the airway wall to investigate the regional differences associated with specific particulate matter, e.g., aerosolized pharmaceutical drugs and pollutants, to the human lung. It remains difficult to study these phenomena in detail using experimental methods in a subjectspecific manner.

In most of previous CFD simulations for lungs, airway geometry was assumed to be rigid due in part to the complexity of geometric modeling and flow simulation methods, availability of 
data, and computational cost. However, in reality, lungs deform and thus, e.g., airway diameter and length vary during breathing. Furthermore, airway deformation could have a stronger influence on airflow in diseased lungs. For example, the variation of trachea diameter is larger in patients with acquired tracheobronchomalacia, resulting in airflow limitation during quiet breathing (Loring et al. (2007)). Therefore, CFD simulations with deforming airway geometry would provide more realistic results.

There are mainly two ways to model deforming lungs: fluid structure interaction (FSI) and imaging-based models. FSI models solve for both tissue deformation and airflow, but they require realistic tissue properties (Wall and Rabczuk (2008), Xia et al. (2008)). In contrast, imaging-based models require prescription of tissue deformation, but they are computationally less expensive than FSI models as they do not solve for tissue deformation (Mead-Hunter et al. (2013), Sera et al. (2013), Yin et al. (2013)). For example, Mead-Hunter et al. (2013) and Sera et al. (2013) deformed their lung models using one image, prescribing the deformation based on the location of arbitrary points relative to a reference point. Yin et al. (2013) used two and three static images to deform their lung models with a sinusoidal waveform, interpolating airway geometry as a function of time.

In order to reproduce lung hysteresis (Mead et al. (1957)), one needs to use images at at least four time points: end expiration, during inspiration, end inspiration, and during expiration. In addition, static images provide quasi-static regional ventilation, which could result in, e.g., unphysiological pressure drop. 4D-dynamic images can be taken at four time points or more per breathing cycle, thus they can provide dynamic regional ventilation. While we proposed a CFD model using three static images (Yin et al. (2013)), using dynamic images in a CFD model is not necessarily a simple extension of the previous model due to more irregular lung deformation. 
Therefore, the main objectives of this study are twofold. First, we aim to develop a CFD model for a deforming human lung using 4DCT images. Second, we investigate the effects of relative hysteresis of lung structure with respect to total lung air volume, airway deformation, and dynamic vs. static imaging methods on pressure drop along the central airways.

In this paper we present new methodologies that allow the application of a CFD model to 4DCT image data. Having established the 4DCT CFD procedure, we apply it to a previously acquired data set of a healthy human volunteer to demonstrate regional divergence of predictions in deforming and rigid airway models when using the full 4D data set vs. multiple combinations of limited time points and statically vs. dynamically acquired CT images.

\section{Methods}

We performed CFD simulations of airflow in deforming and rigid airway models based on 4D dynamic and static CT images. The overall 4DCT CFD procedure is illustrated in Figure 1. The dynamic images were obtained at 13 time points (Figure 1(a)). Deformations of airway surface meshes were obtained prior to CFD simulations by means of an image registration technique (Yin et al (2011)) and the constrained cubic-spline interpolation function (Kruger (2003)) (Figures 1(b)-(e)), whereas airway volume meshes were deformed during simulations using a computational-solid-mechanics (CSM)-based algorithm bounded by the surface meshes. As for airflow boundary conditions, the flow rates at the branches at the distal end of CT-resolved bronchial tree (simply “ending branches" hereafter) were determined in a physiologically realistic manner using an image registration technique and a volume-filling method (Tawhai et al. (2000), Yin et al. (2013)) (Figures 1(b)(c)(f)(g)). Using the above methods, we compared several airway models constructed with dynamic and static images to investigate the effects of 

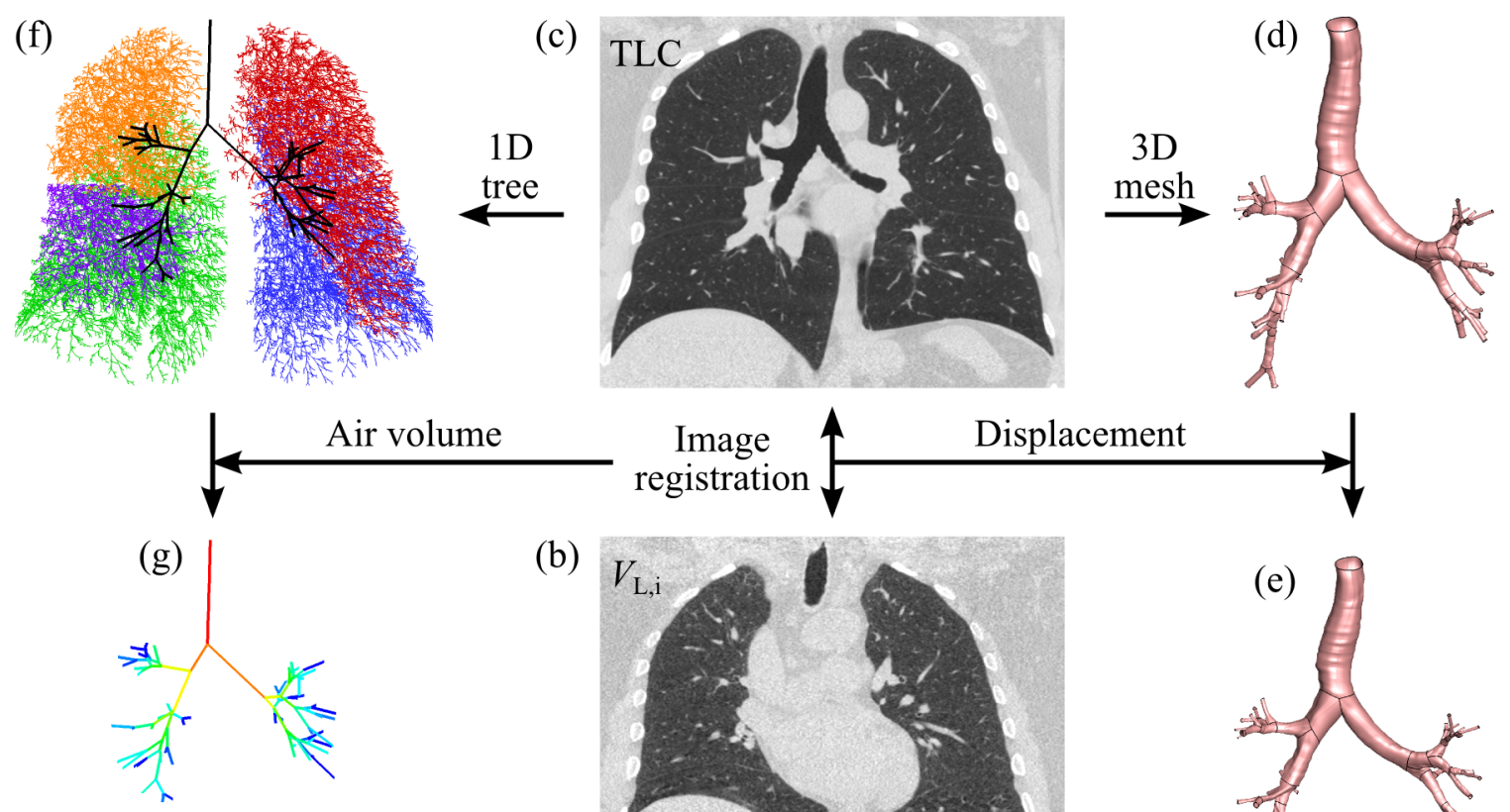

(b)
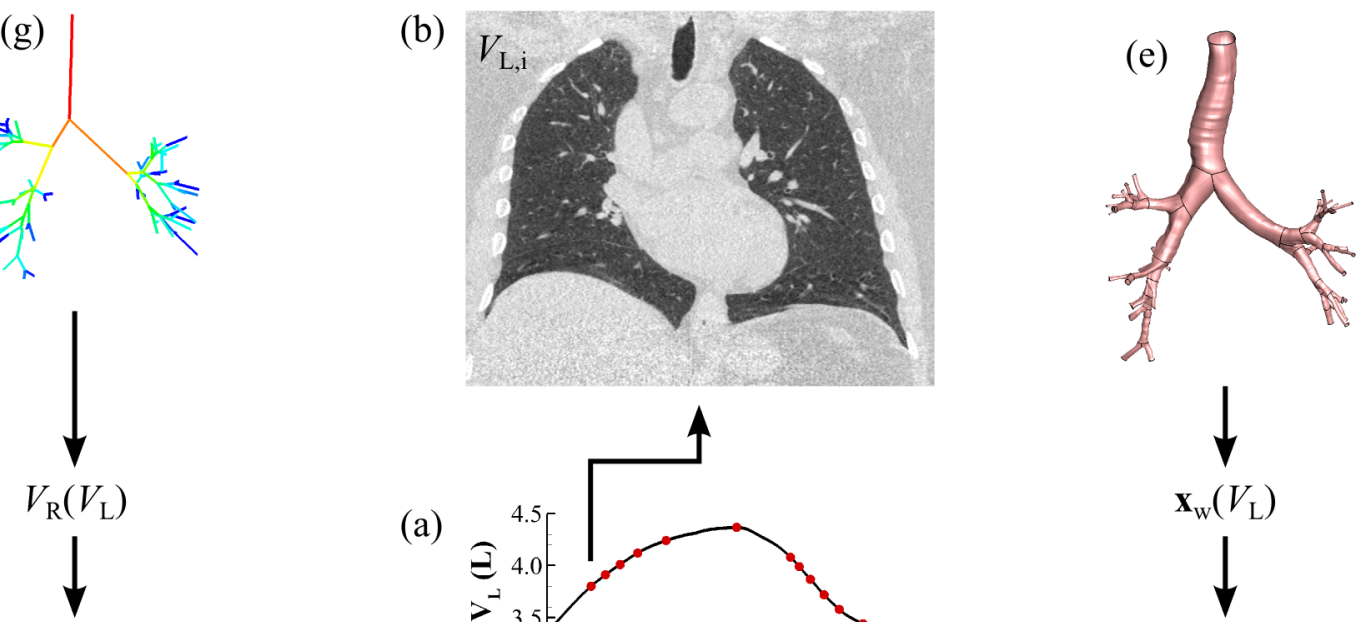

Flow rate at ending branches
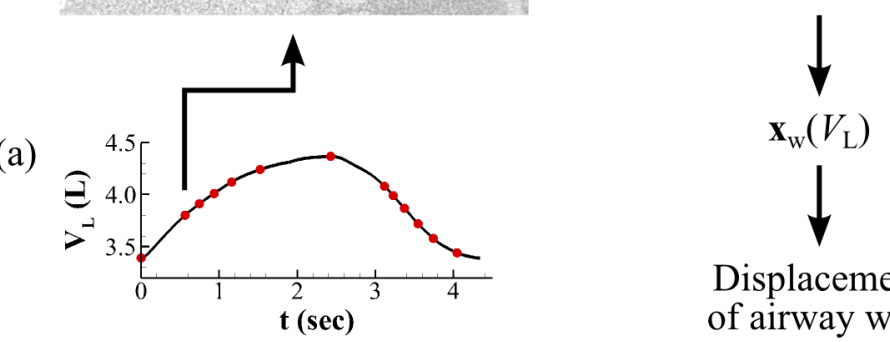

Displacement of airway wall

Figure 1. Summary of the methods: (a) the time history of lung volume $V_{L}(t)$ recorded with a flow meter and the 13 time points for dynamic images (red dots), (b) dynamic image at the $i^{\text {th }}$ time point $t_{i}$, (c) static image at total lung capacity (TLC), (d) airway geometry at TLC, (e) airway geometry at $t_{i}$, (f) 1D tree at TLC, and (g) airflow distribution at $t_{i}$. (b), (e), and (g) were repeated for all 13 time points to obtain regional volume $V_{R}\left(V_{L}\right)$ and airway surface geometry $\mathbf{x}_{\mathrm{W}}\left(V_{\mathrm{L}}\right)$. CT images, $1 \mathrm{D}$ tree, and 3D meshes are not relatively scaled.

relative hysteresis, airway deformation, and imaging method, i.e., dynamic vs. static scans, on the CFD solutions. Each step in Figure 1 is described in more detail below. Specifically, Figures 
1(a)-(c) are described in the subsection 2.1, Figures 1(d)(e) are described in the subsection 2.3, and Figures 1(f)(g) are described in the subsection 2.4.

\subsection{CT image acquisition}

In this study, we developed the numerical methods using 4D-dynamic and static CT lung images, acquired by Janahi et al. (2015), of one of five healthy human subjects. A static image is obtained through conventional spiral scanning while a subject is holding his or her breath, whereas dynamic images are obtained through retrospective reconstruction of multiple point within the tidal volume range obtained from slow pitch spiral scanning during controlled tidal breathing (Jahani et al. (2015)). Therefore, the geometric models based on static and dynamic images may reflect very slow respiratory rates (static acquisitions) and more physiologically meaningful (dynamic) rates, respectively. All subjects were studied in the supine body posture. As for the development of an empirical airflow boundary condition for the rigid airway model based on one static image, we used the data of all five subjects. The imaging protocols and subsequent image assessments here were approved by the Institutional Review Board and the Radiation Safety Committee of the University of Iowa.

The five subjects (Subjects 2-6 in Jahani et al. (2015)) consisted of two females and three males, their ages ranging from 23 to 58 years. A total of 13 volumetric lung images representing 13 time points within the respiratory cycle were reconstructed to capture the realistic deformation of the lung (Figures 1(a)(b)). Tidal breathing was maintained consistent throughout data acquisition via use of a pair of rolling seal pistons (Fuld et al. (2012)). The imaging protocol

and data acquisition were described by Jahani et al. (2015). In addition to dynamic images, static images of the same subjects were obtained at total lung capacity (TLC) and functional residual 


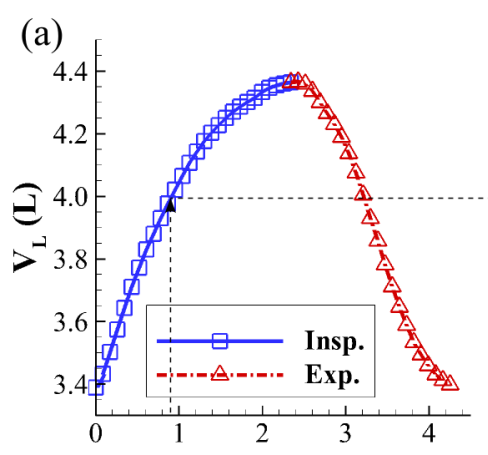

Time (sec)
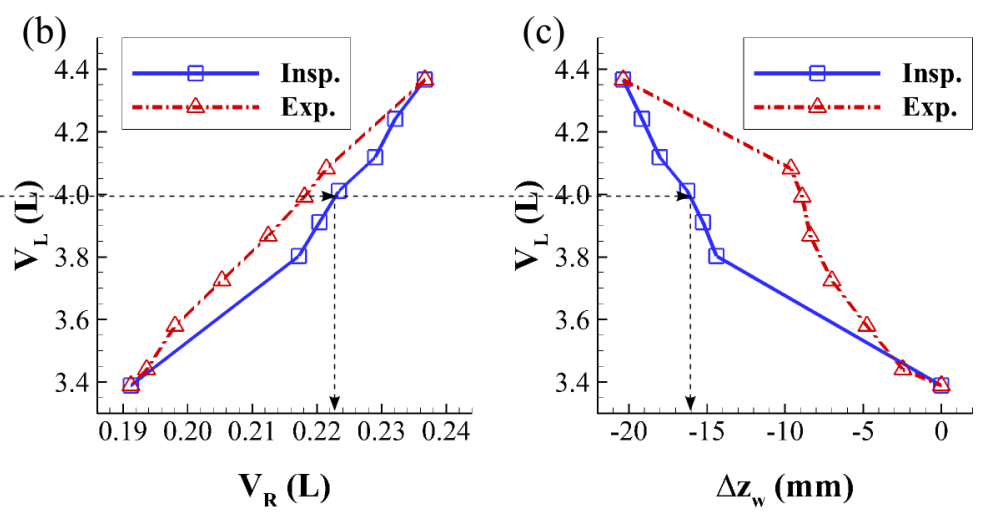

Figure 2. Data points for (a) time-lung volume curve $V_{L}(\mathrm{t})$ of the subject considered, (b) lung volume-regional volume curve $V_{R}\left(V_{L}\right)$ associated with a branch, and (c) lung volume-surface mesh curve $\mathbf{x}_{\mathrm{w}}\left(V_{L}\right)$ (only the displacement along the apical-basal axis relative to EE $\Delta z_{\mathrm{w}}\left(V_{L}\right)$ is shown here) at the end of a branch during inspiration (Insp.) and expiration (Exp.). Because the data in (a) were measured with a turbine-based flow meter and the data in (b) and (c) were derived from the 4DCT images, there are 50 data points in (a) and 13 data points in (b) and (c). The dashed arrows indicate how regional volume and nodal coordinates are computed at an arbitrary time point during the simulations. That is, given a time point, (a) yields $V_{L}$, which produces $V_{R}$ and $\Delta z_{\mathrm{w}}$ by interpolation in (b) and (c).

capacity (FRC) using a pneumotachometer-based volume controller also described by Jahani et al. (2015) (Figure 1(c)).

Using a turbine-based flow meter (Fuld et al. (2012)), Jahani et al. (2015) also recorded the time history of lung volume for the 14 respiratory cycles during which 4DCT image data were acquired. The integrated flow data were used to compute the average breathing period and phase-averaged time-lung volume curve, $V_{L}(t)$ (Figure 1(a)). The average breathing period was $4.34 \mathrm{sec}$, and the phase-averaged $V_{L}(t)$ is shown in Figure 2(a). The lung volumes at end expiration (EE) and end inspiration (EI) were 3.39 and 4.37 L, respectively, so the tidal volume 
Table 1. Summary of simulation cases. Cubic, linear, and no interpolations were used for $\mathbf{x}_{\mathrm{w}}\left(V_{\mathrm{L}}\right)$ of the cases with 13, 2, and 1 images, respectively. "Images for $d V_{R} / d t$ " is the number of images used to compute flow rate at distal end of an ending branch.

\begin{tabular}{cccccc}
\hline Case & 1 & 2 & 3 & 4 & 5 \\
\hline Name & default & LD & RD & LS & RS \\
Image & dynamic & dynamic & dynamic & static & static \\
Images / cycle & 13 & 2 & 1 & 2 & 1 \\
Images for $d V_{R} / d t$ & 2 & 2 & 2 & 2 & 1 \\
\hline
\end{tabular}

of the lung was $0.98 \mathrm{~L}$. The flow rate, trachea hydraulic diameter, and corresponding Reynolds number at peak inspiration (or peak expiration) were $50.0 \mathrm{~L} / \mathrm{min}, 18.9 \mathrm{~mm}$, and 3740 (or 51.8 $\mathrm{L} / \mathrm{min}, 19.0 \mathrm{~mm}$, and 3850), respectively. The lung volumes of static images at TLC and FRC were 6.36 and $2.51 \mathrm{~L}$, respectively. The static image at TLC was used as the reference image for image registration because it resolved more airways as in Yin et al. (2013) (Figure 1(c)).

\subsection{Simulation cases}

We performed CFD simulations for five cases: default, linear dynamic (LD), rigid dynamic (RD), linear static (LS), and rigid static (RS) (Table 1). The dynamic images were used for the first three cases, and the static images were used for the last two cases. The airway surface deformation with respect to lung volume, $\mathbf{x}_{\mathrm{w}}\left(V_{\mathrm{L}}\right)$, was non-linear in the default case and linear in the LD and LS cases, whereas the airway geometry was not deformed, i.e., rigid, in the RD and RS cases. The two images used to construct the airway surface meshes in the linear, LD and LS, cases were those at EE and EI, while the image for the rigid, RD and RS, cases was the one at EI 
(a)

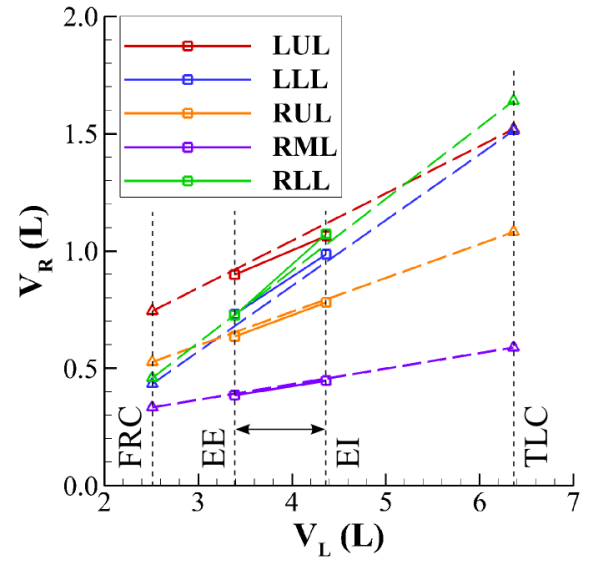

(b)

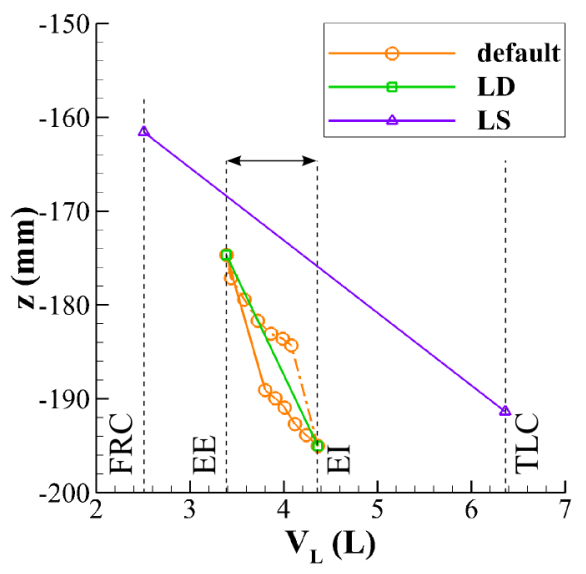

Figure 3. (a) Regional volume $V_{\mathrm{R}}$ of the left upper lobe (LUL), left lower lobe (LLL), right upper lobe (RUL), right middle lobe (RML), and right lower lobe (RLL) for the dynamic (solid) and static (long dashed) cases as a function of lung volume $V_{\mathrm{L}}$. (b) z-coordinate of a node in the RLL during inspiration (solid) and expiration (dash-dotted) for the default, LD, and LS cases as a function of $V_{\mathrm{L}}$. Black dashed lines indicate FRC, EE, EI, and TLC, and black arrows indicate the range of $V_{\mathrm{L}}$ during the multiple-image simulations.

(Figure 3). The static images at EE and EI were obtained by linear interpolation between the images at FRC and TLC with respect to lung volume.

In this study, we aimed to investigate the effects of relative hysteresis, lung deformation, and imaging methods on CFD solutions. First, we used the default and LD cases to investigate the effect of relative hysteresis on CFD solutions. Second, we compared the four cases LD, RD, LS, and RS against the default case to assess the effect of lung deformation. Finally, we compared the LD and LS cases to estimate the effect of imaging methods: dynamic vs. static scans because dynamic and static images yielded airway geometry of different size even at the same lung volume. 

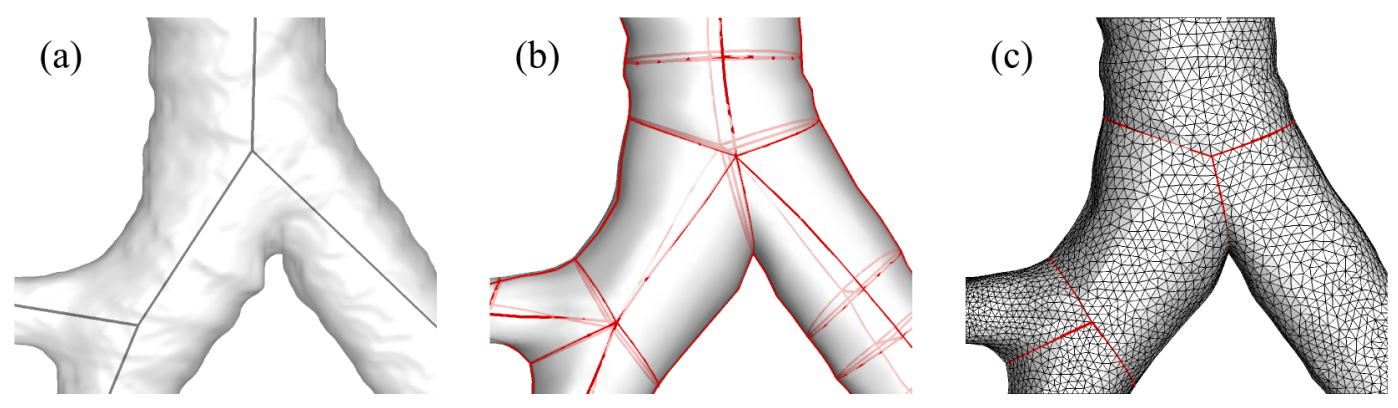

Figure 4. (a) Airway skeleton and airway wall geometry extracted from a CT image, (b) 3D airway geometry extruded from the skeleton and fitted to the extracted airway wall geometry, and (c) 3D surface mesh of the airway for CFD.

\subsection{CFD mesh generation and mesh sensitivity test}

We constructed the airway geometry from the reference image (at TLC in this study) and generated the CFD mesh using the geometric modeling and meshing methods by Miyawaki et al. (2013, 2014) (Figures 1(c)(d)). The generated CFD mesh consisted of 108 branches, including 56 ending branches. In these methods, the 3D surface geometry is extruded from the skeleton of the airway, the surface geometry is fitted to the airway wall geometry extracted from the image, the grid size is non-uniform in radial and axial directions of the airway branches, and the airway volume is filled with tetrahedral elements (Figure 4). A unique feature of the resulting CFD mesh is that the domain is partitioned into sub-domains corresponding to each branch as outlined in red in Figure 4(c), thus each sub-domain is associated with anatomical information, e.g., generation number and average diameter, which facilitates pre- and post-processes for CFD simulations. Then, we transformed the reference surface mesh to those at different time points using a mass-preserving image registration technique (Yin et al., (2011, 2013)) (Figures 1(a)(e)). 
In addition to the five cases in Table 1, we coarsened and refined the mesh for the default case to perform the mesh sensitivity test. The two cases were referred to as the coarse and fine cases. The average distances from the wall to the first grid at peak inspiration were 11.4, 7.5, and 5.7 in wall unit for the coarse, default, and fine cases, respectively. In addition, the numbers of tetrahedral elements for the coarse, default, and fine cases were 1.5, 5.3, and 11.7 million, respectively. The result of the mesh sensitivity test showed that the difference in pressure drop between the default and fine cases was three times smaller than that between the default and coarse cases (3.9\% vs. $12.2 \%)$. Thus, the mesh size in the default case was adequate for this study and it was used for the other cases: LD, RD, LS, and RS (Table 1).

\subsection{Boundary conditions}

We imposed the following boundary conditions to solve for air velocity and pressure fields:

1. A cross-section proximal to the glottal constriction (which is treated as an inlet during inspiration and an outlet during expiration): (a) time-varying uniform velocity profile with synthetic turbulence during inspiration and (b) time-varying parabolic velocity profile during expiration

2. Distal ends of ending branches (outlets during inspiration and inlets during expiration): time-varying parabolic velocity profile

In addition, deforming airway surface mesh velocity derived from image registration was imposed on all boundaries. Uniform or parabolic velocity profiles with known cross-sectional areas were imposed to prescribe flow rates. To solve for airway volume mesh velocity, we imposed displacement derived from image registration on all boundaries. 
We attached a physiologically-consistent laryngeal model to the top of the trachea to produce a turbulent laryngeal jet (Miyawaki et al. (2013)). As the flow above the glottal constriction is usually turbulent (Choi et al. (2010)), we fed synthetic turbulence above the glottal constriction during inspiration using a synthetic-eddy-method (Jarrin et al. (2006)). The turbulent kinetic energy distribution in the trachea and the particle distribution in the central airways predicted with the laryngeal model were consistent with those predicted with the CTbased extrathoracic model (Miyawaki et al. (2013)).

To determine the flow rate at the distal end of each CT-resolved ending branch, we defined the regional volume $V_{R}$ as the volume of the peripheral region to which the ending branch ventilates (Figure 5). Lung parenchyma was partitioned into 30,000 units that are then directly linked to the modeled terminal bronchioles, and air volume in each lung parenchymal unit (approximately the size of an acinus) was obtained by assessment of the regional Hounsfield Unit (Yin et al. (2013)) (Figure 1(c)). We modeled the full airway tree in the region where airways were not resolved by CT imaging with a volume-filling technique to link ending branches with lung parenchymal units (Tawhai et al. (2000)) (Figures 1(c)(f); artificial airways are color-coded by lobe in Figure 1(f)). We then obtained the $V_{R}$ for the ending branches in the reference image by adding up air volume in lung parenchymal units with the aid of the artificial airway tree (Figures 1 (c)(f)). For example, the volume of the red dashed region in Figure 5 is the $V_{R}$ for the red ending branch, and this region is filled with the artificial airway tree extended from the red ending branch. Similarly, the volume of the blue dashed region in Figure 5 is the $V_{R}$ for the blue branch, and this region is filled with the artificial airway trees extended from the ending branches a, b, and c. $V_{R}$ at different time points can be obtained by image registration (Figures 1(a)(b)(c)(f)(g)). 


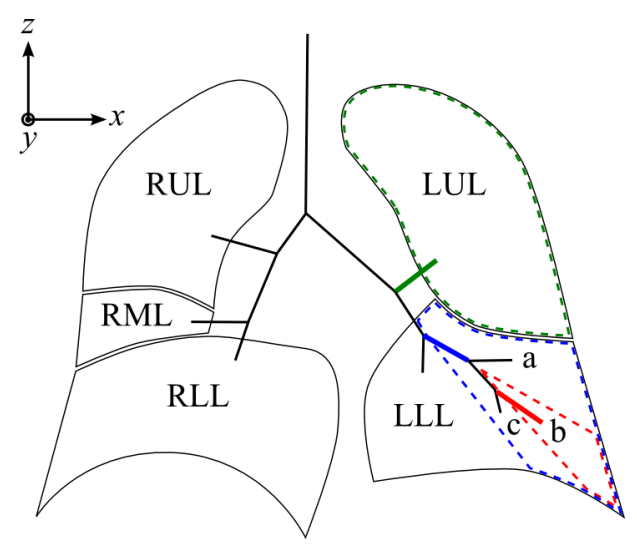

Figure 5. Definitions of coordinate system and regional volume $V_{R} \cdot y$-direction points toward the paper. A colored branch ventilates the region surrounded by the dashed curves with the same

To simplify the time-varying boundary condition, we decomposed $V_{R}(t)$ into $V_{R}\left(V_{L}\right)$ and $V_{L}(t)$ (Figures 2(a)(b)). This allows one to investigate the relative hysteresis of $V_{R}$ with respect to $V_{L}$, i.e., the difference between inspiration and expiration in $V_{R}\left(V_{L}\right)$ (Froeb and Mead (1968)). The time rate of the change of regional volume $d V_{R} / d t$ gives the flow rate at an ending branch. By chain rule, the flow rate was calculated as follows:

$\frac{d V_{R}}{d t}=\frac{d V_{R}}{d V_{L}} \frac{d V_{L}}{d t}$

where $d V_{L} / d t$ is the total flow rate at the mouth or in the trachea, and $d V_{R} / d V_{L}$ is the fraction of the total flow rate distributed to an ending branch. Similarly, we obtained the deforming airway surface mesh via $\mathbf{x}_{\mathrm{w}}\left(V_{L}\right)$ and $V_{L}(t)$ (Figures 2(a)(c)), where the $x$-, $y$-, and $z$-directions are positive to the left, backward (dorsal), and upward (cephalad), respectively, as shown in Figure 5.

To obtain lung volume, regional volume, and surface mesh at an arbitrary time or lung volume during CFD simulations (following the dashed arrows in Figure 2), we constructed interpolation functions for $V_{L}(t), V_{R}\left(V_{L}\right)$, and $\mathbf{x}_{\mathrm{w}}\left(V_{L}\right)$. Note that the number of data points for $V_{L}(t)$ 
was much more than those for $V_{R}\left(V_{L}\right)$ and $\mathbf{x}_{\mathrm{w}}\left(V_{\mathrm{L}}\right)$ because the former was measured with a turbine-based flow meter and the latter was acquired from a CT scanner. Since $V_{L}(t)$ is a convex smooth curve, we constructed the cubic-spline function (Press et al. (2007)) using 29 and 23 data points for inspiration and expiration, respectively, with zero-gradient boundary condition (Equation S.1 in Supplementary Material). As will be discussed later, the relative hysteresis in $V_{R}\left(V_{L}\right)$ was small, exhibiting almost linear relationship. Thus, $V_{R}\left(V_{L}\right)$ was approximated as a linear function between the $V_{R}$ values at EE and EI. In contrast, the relative hysteresis in airway geometry $\mathbf{x}_{\mathrm{w}}\left(V_{L}\right)$ was not small, so the constrained cubic-spline function (Kruger (2003)) was constructed using 7 and 8 data points for inspiration and expiration, respectively, with natural, zero curvature, boundary condition (Equation S.2 in Supplementary Material).

For rigid airway models based on one static image at EI, $d V_{\mathrm{R}} / d V_{\mathrm{L}}$ in Equation 1 has to be approximated. We propose an empirical boundary condition based on the correlation between the fraction of the regional volume at TLC, $v_{\mathrm{R}, \mathrm{TLC}}$, and the fraction of the difference between the regional volumes at TLC and FRC, $\Delta v_{\mathrm{R}}$, for ending branches (Figure 6(a)).

$\Delta v_{\mathrm{R}}=0.901( \pm 0.136) v_{\mathrm{R}, \mathrm{TLC}}{ }^{0.972( \pm 0.040)}(r=0.972( \pm 0.008))$

where the parameters are presented as means ( \pm standard deviation) based on five healthy subjects and $r$ is the correlation coefficient. $v_{\mathrm{R}, \mathrm{TLC}}$ and $\Delta v_{\mathrm{R}}$ for the ending branch $e$ are defined as

$$
\begin{aligned}
& V_{R, T L C, e}=\frac{V_{R, T L C, e}}{\sum_{i=1}^{N_{e}} V_{R, T L C, i}} \\
& \Delta v_{R, e}=\frac{V_{R, T L C, e}-V_{R, F R C, e}}{\sum_{i=1}^{N_{e}}\left(V_{R, T L C, i}-V_{R, F R C, i}\right)} \approx \frac{d V_{R, e}}{d V_{L}}
\end{aligned}
$$



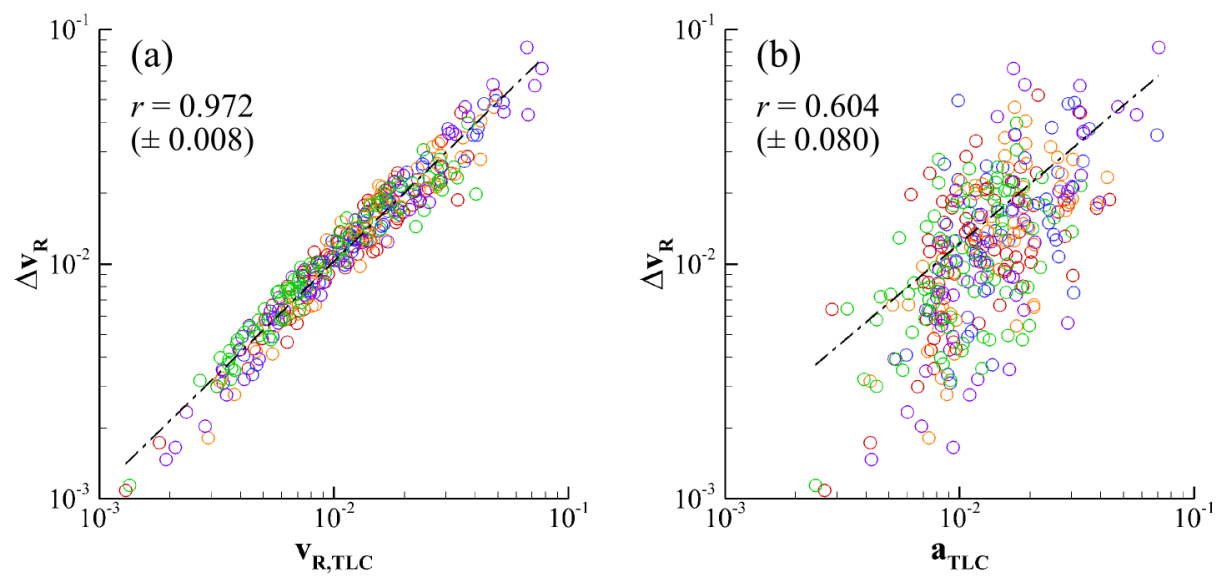

Figure 6. Correlation (a) between fraction of regional volume at TLC, $v_{\mathrm{R}, \mathrm{TLC}}$, and fraction of regional volume difference $\Delta v_{R}$ and (b) between luminal area fraction at TLC, $a_{\mathrm{TLC}}$, and $\Delta v_{\mathrm{R}}$ for ending branches of five healthy subjects including this subject colored by subject. Each dot corresponds to an ending branch, and $r$ denotes correlation coefficient. The equations of the regression curves were (a) $\Delta v_{\mathrm{R}}=0.901( \pm 0.136) v_{\mathrm{R}, \mathrm{TLC}}{ }^{0.972( \pm 0.040)}(r=0.972( \pm 0.008))$ and (b) $\Delta v_{\mathrm{R}}=0.591( \pm 0.499) a_{\mathrm{TLC}}{ }^{0.842( \pm 0.176)}(r=0.604( \pm 0.080))$, where the parameters are presented as mean \pm (standard deviation).

where $V_{\mathrm{R}, \mathrm{TLC} \text {, }}$ and $V_{\mathrm{R}, \mathrm{FRC}, \mathrm{e}}$ are the $V_{\mathrm{R}}$ of the branch $e$ at TLC and FRC, respectively, and $N_{\mathrm{e}}$ is the number of ending branches for each subject. Since the change in airway volume was less than $1 \%$ of the change in lung volume, $d V_{\mathrm{R}} / d V_{\mathrm{L}} \approx \Delta v_{\mathrm{R}}$. Because the correlation coefficient, the coefficient in the regression equation, and the power in the regression equation were all close to unity, i.e., $\Delta v_{\mathrm{R}} \approx v_{\mathrm{R}, \mathrm{TLC}}$, we propose to use $v_{\mathrm{R}, \mathrm{TLC}}$ and approximate $d V_{\mathrm{R}} / d V_{\mathrm{L}}\left(\approx \Delta v_{\mathrm{R}} \approx v_{\mathrm{R}, \mathrm{TLC}}\right)$ in Equation 1 in calculation of the flow rates at ending branches.

To prescribe flow rate above the glottal constriction, we used the breathing waveform $V_{L}(t)$ measured during the dynamic scanning for all five cases (Table 1). We used the same $V_{L}(t)$ 
to compute the flow rate at distal ends of ending branches in Equation 1. Except the RS case, the $V_{R}\left(V_{L}\right)$ was a linear function varying between the $V_{\mathrm{R}}$ values at EE and EI, as will be discussed in detail later. Note the flow rates for the cases based on dynamic images are the same (Table 1). For the RS case, the $d V_{\mathrm{R}} / d V_{\mathrm{L}}$ was set to $v_{\mathrm{R}, \mathrm{TLC}}$ because of the approximation of $d V_{\mathrm{R}} / d V_{\mathrm{L}} \approx \Delta v_{\mathrm{R}} \approx$ $v_{\mathrm{R}, \mathrm{TLC}}$ based on Equation 2. Note $v_{R, \mathrm{EI}} \approx v_{R \text {,TLC }}$ because $d V_{R} / d V_{L}$ was almost linear. It is also worth noting that even if lung volumes for dynamic and static cases were the same, the regional volumes and surface meshes were different (Figure 3).

\subsection{CFD with deforming mesh}

We used a large eddy simulation (LES) model and the arbitrary Lagrangian-Eulerian (ALE) method to simulate laminar, transitional, and turbulent airflows with moving mesh as in Yin et al. (2013):

$\nabla \cdot \mathbf{u}=\mathbf{0}$

$$
\frac{\partial \mathbf{u}}{\partial t}+(\mathbf{c} \cdot \nabla) \mathbf{u}=-\frac{1}{\rho} \nabla p+\nabla \cdot\left\{\left(v+v_{T}\right)\left[\nabla \mathbf{u}+(\nabla \mathbf{u})^{T}\right]\right\}
$$

where $\mathbf{u}$ is the fluid velocity, $t$ is time, $\mathbf{c}=\mathbf{u}-\hat{\mathbf{u}}$ is the convective velocity, $\hat{\mathbf{u}}$ is the mesh velocity, $\rho$ is the fluid density, $v$ is the fluid kinematic viscosity, and $v_{T}$ is the subgrid-scale eddy viscosity (Vreman (2004)), which reduces to zero for laminar flow. The $\rho$ and $v$ of air were 1.2 $\mathrm{kg} / \mathrm{m}^{3}$ and $1.5 \times 10^{-5} \mathrm{~m}^{2} / \mathrm{s}$, respectively.

To deform the volume mesh, we employed a CSM-based algorithm (Stein et al. (2003)) by imposing the displacement of the surface meshes between two consecutive time steps as essential boundary condition. We used a non-linear formulation for large deformation, and the Lagrangian form of the balance of linear momentum reads: 
$\operatorname{div} \mathbf{P}+\rho_{0} \mathbf{b}=\mathbf{0}$

where $\mathbf{P} \boldsymbol{\sigma} \mathbf{F} J^{-T}$ is the first Piola-Kirchhoff stress, $\boldsymbol{\sigma}$ is the Cauchy stress, $\mathbf{F} \boldsymbol{(} \mathbf{F} \partial \mathbf{X} \partial$ is the

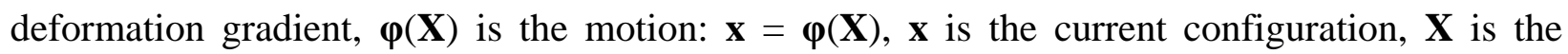
reference configuration, $J=\operatorname{det} \mathbf{F}$ is the Jacobian, $\rho_{0}$ is the density in the reference configuration, and $\mathbf{b}$ is the body force. We used the St. Venant Kirchhoff material:

$\mathbf{S}=\lambda(\operatorname{tr} \mathbf{E}) \mathbf{I}+2 \mu \mathbf{E}$

where $\mathbf{S}=\mathbf{F}^{-1} \mathbf{P}$ is the second Piola-Kirchhoff stress, $\mathbf{E}$ is the Green-Lagrange strain, $\mathbf{I}$ is the identity tensor, and $\lambda$ and $\mu$ are Lamé constants. Stein et al. (2003) adjusted the ratio of $\left(\lambda+2 \mu / n_{s d}\right)$ and $2 \mu$ to stiffen the mesh against volume or shape change, where $n_{s d}$ is the number of space dimensions. They also replaced $J$ with $J\left(J^{0} / J\right)^{\chi}$ to stiffen smaller elements, where $\chi(\geq 0)$ is the stiffening parameter, and $J^{0}$ is an arbitrary scaling parameter for dimensional consistency. In this study, the ratio of $\left(\lambda+2 \mu / n_{s d}\right)$ and $2 \mu$ was $0.5, \chi$ was 0.7 , and $J^{0}$ was 1.0 .

The procedure in the deforming lung CFD model is summarized as follows:

1. At an arbitrary time, compute lung volume $V_{\mathrm{L}}(t)$ using the cubic-spline function (Figure 2(a)).

2. Compute surface mesh displacement $\mathbf{x}_{\mathrm{w}}\left(V_{\mathrm{L}}\right)$ using the constrained cubic-spline function (Figures 2(c)).

3. Compute volume mesh displacement $\mathbf{x}\left(V_{\mathrm{L}}\right)$ and velocity $\hat{\mathbf{u}}$ by solving Equation 7 with prescribed boundary displacement.

4. Compute flow rate $d V_{R} / d t$ at the distal end of ending branches using Equation 1 . 
5. Compute air velocity $\mathbf{u}$ and pressure $p$ by solving Equations 5 and 6 with flow rate prescribed at the distal end of ending branches.

\subsection{Quantitative analysis}

To measure the degree of the relative hysteresis of variable $\phi$ with respect to lung volume $V_{\mathrm{L}}$, we defined the average absolute relative hysteresis $h_{\phi}$ as the average difference between the $\phi$ values at the same $V_{\mathrm{L}}$ on the inspiration and expiration curves:

$h_{\phi}=\frac{1}{V_{L, E I}-V_{L, E E}} \int_{V_{L, E E}}^{V_{L, E I}}\left|\phi_{I n}-\phi_{E x}\right| d V_{L}$

where $V_{\mathrm{L}, \mathrm{EI}}$ is $V_{\mathrm{L}}$ at $\mathrm{EI}, V_{\mathrm{L}, \mathrm{EE}}$ is $V_{\mathrm{L}}$ at $\mathrm{EE}, \phi_{\mathrm{In}}$ is $\phi$ during inspiration, and $\phi_{\mathrm{Ex}}$ is $\phi$ during expiration. In this study $\phi$ is $V_{\mathrm{R}}$ or $\mathbf{x}$.

We predicted the pressure drop from the top of the trachea to the distal end of each branch at peak inspiration to quantify the effects of relative hysteresis, airway deformation, and imaging method, because one of the most physiologically important quantities in the assessment of regional patterns of airflow within the lung is pressure drop. To assess the bulk difference in pressure drop between the default and other cases, we first normalized the absolute difference by the value for the default case branch by branch, and averaged the normalized absolute difference over branches:

$\varepsilon_{\Delta p}=\frac{1}{N_{b}} \sum_{b=1}^{N_{b}} \frac{\left|\Delta p_{b}-\Delta p_{b, 0}\right|}{\Delta p_{b, 0}}$

where $N_{b}=52$ is the number of branches considered in the analysis, $\Delta p_{b}$ is the pressure drop from the top of the trachea to the distal end of the branch $b$, and $\Delta p_{b, 0}$ is the $\Delta p_{b}$ for the default 
case. There were 108 branches, including 56 ending branches. In the analysis the ending branches were not considered to minimize the effect of boundary conditions imposed at their distal ends. We also computed average normalized signed difference to evaluate the overall bias compared to the default case:

$\beta_{\Delta p}=\frac{1}{N_{b}} \sum_{b=1}^{N_{b}} \frac{\Delta p_{b}-\Delta p_{b, 0}}{\Delta p_{b, 0}}$

The major factors that affect the pressure drop include airway cross-sectional area, airway length, airway volume, and flow rate in each branch. Thus we also computed the average differences of these quantities at peak inspiration against the default case. The cross-sectional area was computed at the distal end of each branch, and the length was computed by dividing the volume of the same branch by the cross-sectional area.

\section{Results}

\subsection{Uncertainty in dynamic imaging and linearity in relative hysteresis}

In order to estimate the uncertainty in regional volume derived from dynamic images, we compared regional volumes of two images having similar lung volumes: 1) the image at EI, corresponding to the right ends of the curves in Figure 7, and 2) the image with $0.3 \%$ smaller lung volume than the one at EI. Because the regional volumes of two close lung volumes are expected to be similar, the difference between them was used as a measure of the uncertainty in dynamic imaging (O’Connel et al. (2015)). The difference between the regional volumes of the two images, $\Delta V_{\mathrm{R}, \mathrm{EI}}=\left|V_{\mathrm{R}, \mathrm{EI}}-V_{\mathrm{R}, \mathrm{EI}-0.3 \%}\right|$, was then compared against the average relative hysteresis

$h_{V_{R}}$ branch by branch (Equation 9). If the normalized difference $\Delta V_{R, E I} / h_{V_{R}}$ is close to unity, the uncertainty from dynamic imaging is comparable to the relative hysteresis. The result showed 

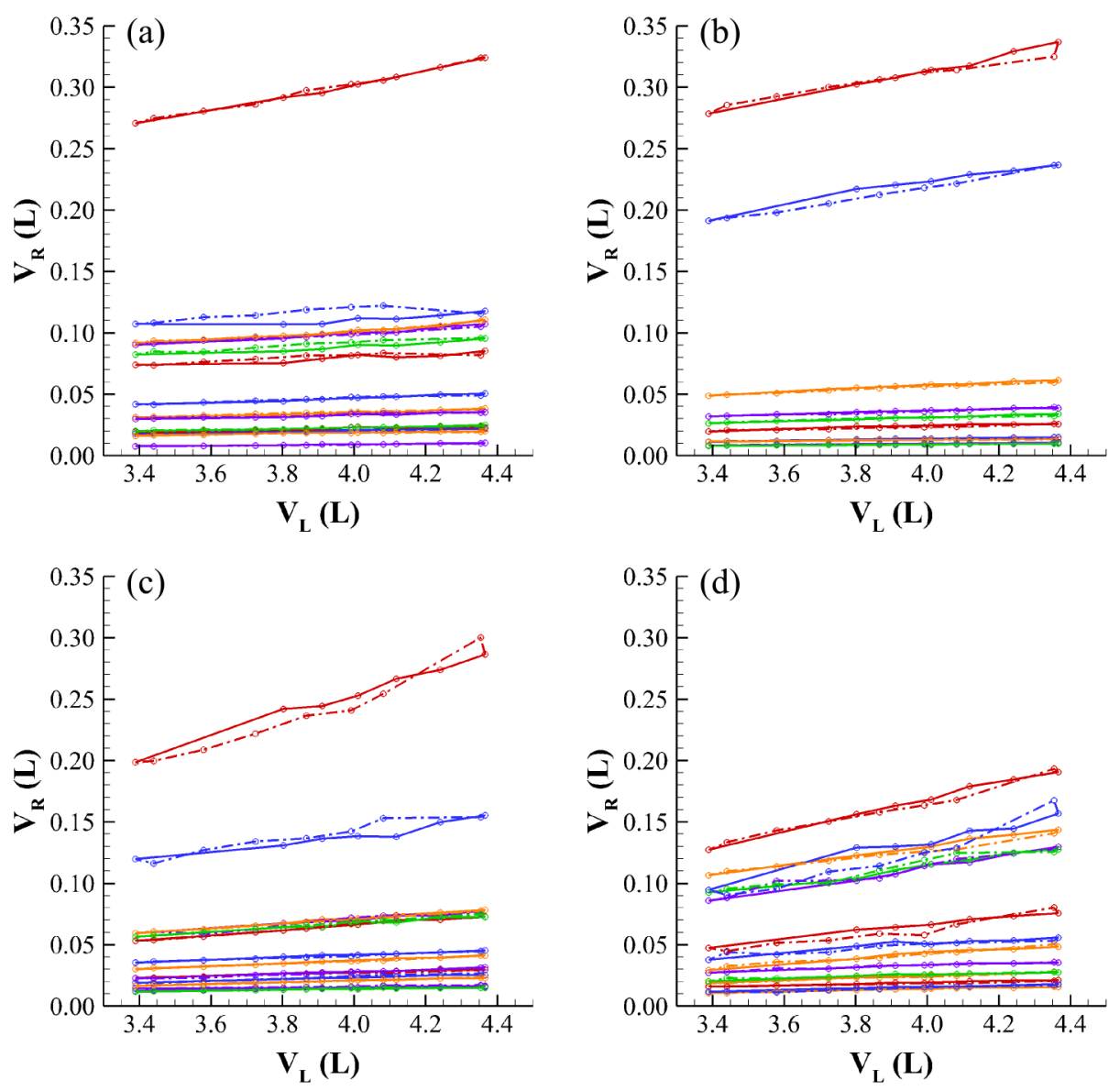

Figure 7. Relative hysteresis of regional volumes $V_{\mathrm{R}}$ with respect to lung volume $V_{\mathrm{L}}$ during inspiration (solid) and expiration (dashed-dot): regions connected to 56 ending branches in (a) the left upper lobe, (b) right upper lobe, (c) left lower lobe, and (d) right lower lobe. The plots include one additional data point near end inspiration during expiration.

that $\Delta V_{R, E I} / h_{V_{R}}$ was $1.63( \pm 1.45)$ on average for 56 ending branches. That is, the uncertainty was $63 \%$ larger than the relative hysteresis of regional volume for ending branches. This implied that the relative hysteresis of regional volume was smaller than the difference between the regional volumes of two images close to each other near EI. We then repeated the same analysis for the relative hystereses in the $x-, y$-, and $z$-displacements of the deforming airway geometry, 
e.g., the $z_{w}\left(V_{L}\right)$ relationship as shown in Figure 8. The normalized differences between the displacements of the two images were $\Delta x_{E I} / h_{x}=0.95( \pm 0.79), \Delta y_{E I} / h_{y}=0.26( \pm 0.27)$, and $\Delta z_{E I} / h_{z}=0.57( \pm 0.48)$ on average for 52 branches. Thus, the relative hysteresis of displacement with respect to lung volume is more evident than regional volume. Conversely, the uncertainty has less effect on displacement than on regional volume.

To further assess the linearity of the relations $V_{R}\left(V_{L}\right)$ and $z_{w}\left(V_{L}\right)$, we performed the Ramsey RESET test (Ramsey (1969)) for quadratic or cubic influence. Because the relative hysteresis for $V_{R}\left(V_{L}\right)$ in Figure 7 was small, the data for $V_{R}$ during inspiration and expiration were combined when performing the test. Table 2 shows that for $86 \%$ of all ending branches, quadratic or cubic influence was not significant $(p>0.05)$, implying a linear $V_{R}\left(V_{L}\right)$ relationship. The RML had the lowest value of $67 \%$ because there were only three ending branches in this lobe with one of them exhibiting non-linearity. Thus, we used the linear $V_{R}\left(V_{L}\right)$ relationship, resulting in a constant $d V_{R} / d V_{L}$ for each ending branch regardless of time point, that is, the distribution of airflow to ending branches remained constant during CFD simulations.

On the other hand, as for the relative hysteresis in airway surface deformation, only $27 \%$ and $50 \%$ of branches exhibited linearity during inspiration and expiration, respectively, as a result of the linearity test on the $z\left(V_{L}\right)$ relationship as shown in Table 3. More specifically, for most of branches in the right lung quadratic or cubic influence was significant $(p<0.05)$ despite that most of branches in the left lung exhibited linear behaviors during exhalation. Therefore, we used a non-linear interpolation function to obtain $\mathbf{x}_{\mathrm{w}}\left(V_{L}\right)$ for airway surface meshes during the simulations. 

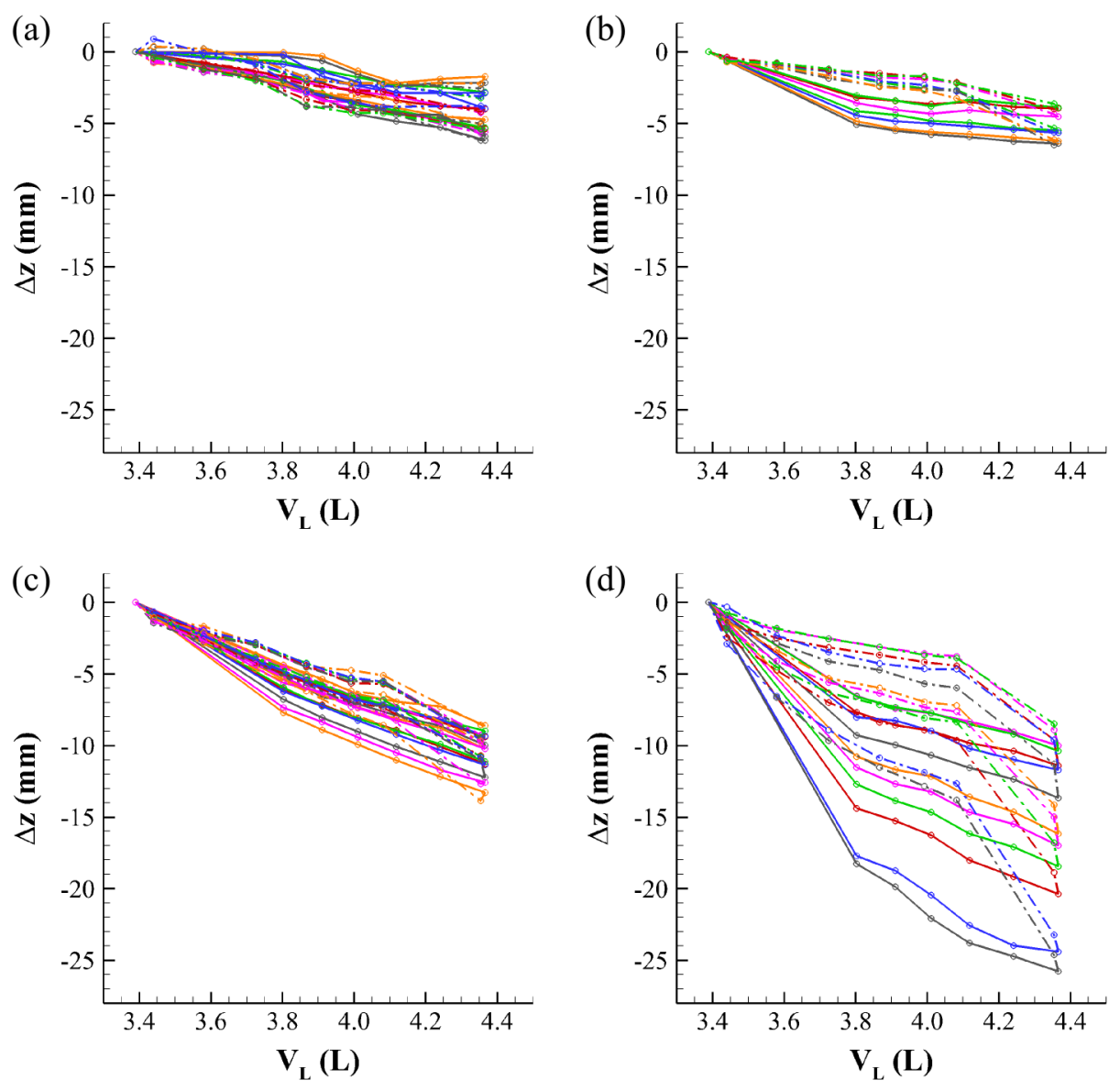

Figure 8. Relative hysteresis of $z$-displacement relative to EE, $\Delta z$, with respect to lung volume $V_{\mathrm{L}}$ during inspiration (solid) and expiration (dashed-dot): distal ends of branches in the (a) left upper lobe, (b) right upper lobe, (c) left lower lobe, and (d) right lower lobe. The plots include one additional data point near end inspiration during expiration.

\subsection{Relative hysteresis in deforming airway and its effect on pressure drop}

To investigate the relative hysteresis of deforming airway geometry with respect to lung volume and its effect on overall pressure drop and velocity field around the first bifurcation, we compared the default and LD cases. The average relative hystereses in $x$-, $y$-, and $z$ displacements of airway surface meshes for the branches in the default case were $0.52,1.53$, and 
Table 2. Results from the Ramsey RESET test for quadratic or cubic influence on $V_{\mathrm{R}}\left(V_{\mathrm{L}}\right)$ and $z\left(V_{\mathrm{L}}\right)$. The values are presented as \% of 56 ending branches (for $V_{\mathrm{R}}$ ) or \% of 52 branches (for $z$ ) for which quadratic or cubic influence was not significant $(p>0.05) . V_{\mathrm{R}}$ during inspiration and expiration were merged because their difference was small. $z_{\mathrm{In}}$ and $z_{\mathrm{Ex}}$ are $z$ during inspiration and expiration, respectively.

\begin{tabular}{cccc}
\hline & $V_{\mathrm{R}}$ & $Z_{\text {In }}$ & $z_{\mathrm{Ex}}$ \\
\hline All lobes & 86 & 27 & 50 \\
LUL & 79 & 69 & 77 \\
LLL & 93 & 14 & 93 \\
RUL & 90 & 0 & 14 \\
RML & 67 & 0 & 0 \\
RLL & 86 & 0 & 0 \\
\hline
\end{tabular}

$1.45 \mathrm{~mm}$, respectively. The relative hystereses in $z\left(V_{L}\right)$ and $y\left(V_{L}\right)$ were about three times greater than that in $x\left(V_{L}\right)$. Thus, the major hysteresis existed in the sagittal $y$-z plane along the dorsalventral $(y)$ axis and the apical-basal $(z)$ axis. The direction of $y\left(V_{L}\right)$ was clockwise for all branches, and that of $z\left(V_{L}\right)$ was counter-clockwise in 73\% of branches, as illustrated in Figure 9. The clockwise feature in $y\left(V_{L}\right)$ indicates that airway wall deformation in the $y$ direction exhibits less hysteresis than lung volume with respect to intrapleural pressure, and vice versa for $z$ direction (Froeb and Mead (1968)). Nonetheless, the difference in airway geometry, measured by the average of the differences in area and length, was only about $1.6 \%$ and the bias based on the signed difference was small (see the rows “Area” and “Length” and column "LD” in Table 3).

The CFD results showed that the difference in pressure drop between the default and LD cases was 1.3 percentage points larger than the difference between the default and fine case 
(5.2\% vs. 3.9\%), and the LD case slightly under-estimated the pressure drop compared with the default case $(-1.9 \%$ vs. $-0.5 \%)$. The airway models for the default and LD cases were about the same size (Figure 10(a)). In addition, there was no clear qualitative difference in 3D flow structure at the first bifurcation between the default vs. LD cases (Figures 11(a)(b)). The differences in bulk velocity were $2.5 \%$ and $1.5 \%$ at the distal ends of the left main bronchus (LMB) and right main bronchus (RMB), respectively, and respective differences in kinetic energy correction factors based on instantaneous velocity fields were $0.7 \%$ (1.51 vs. 1.50) and $4.8 \%$ (1.27 vs. 1.33$)$.

\subsection{Compliant vs. rigid airway models}

The comparisons between the LD and RD cases (based on dynamic images) and between the LS and RS cases (based on static images) were made to investigate the effect of airway deformation. The differences in pressure drop were 8.9 and 21.4 percentage points for the LD vs. RD cases and the LS vs. RS cases, respectively (5.2\% vs. $14.1 \%$ and $29.2 \%$ vs. $50.6 \%$ in Table 3 ). The rigid cases overall under-estimated the pressure drop as shown in the average normalized signed differences in Table 3. The differences in geometry (the average of the differences in area and length) were about 4.3 and 0.4 percentage points for LD vs. RD cases and LS vs. RS cases, respectively. The geometries for the rigid cases were overall larger based on the average normalized signed differences (cf. Figures 10(a) vs. 10(b) and Figures 10(c) vs. 10(d)).

In some CFD simulations similar to the RS case but without data for air-volume-change $\left(d V_{R} / d V_{L}\right)$ (e.g., Case II in Yin et al. (2013)), a uniform velocity boundary condition was imposed. As a result, the flow rate at an ending branch depends on its cross-sectional area. To 

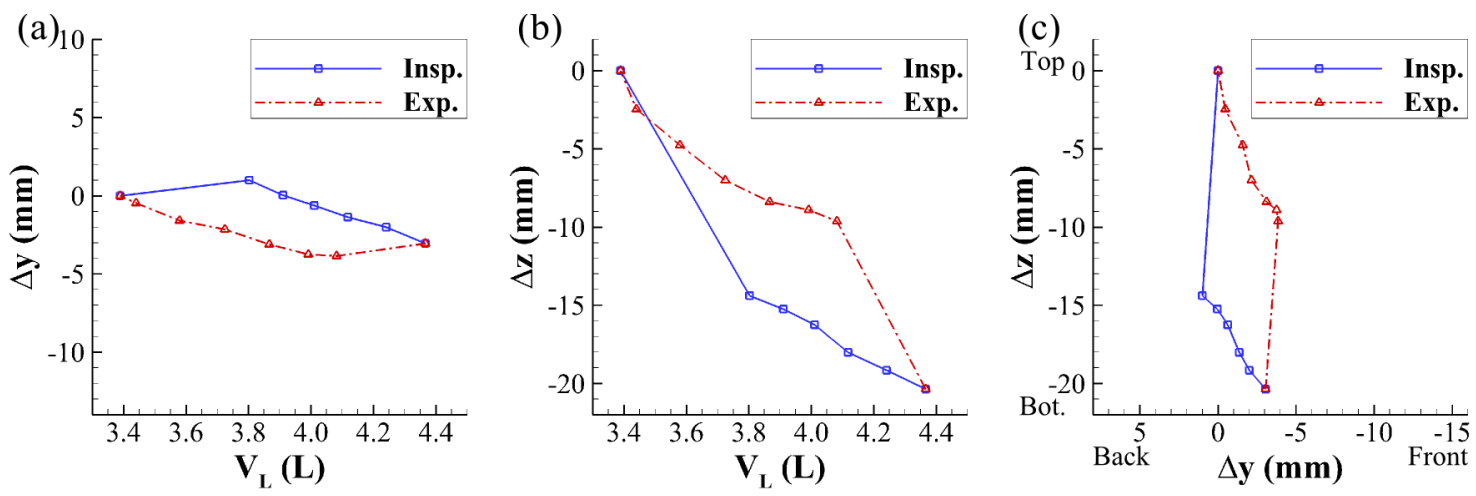

Figure 9. (a) $y$ - and (b) z-displacements, relative to EE, of a branch in the right lower lobe with respect to lung volume $V_{\mathrm{L}}$ during inspiration (Insp.) and expiration (Exp.). (c) Path of the branch during the breathing cycle. The diameter of the branch was 3.35 and $3.41 \mathrm{~mm}$ at end expiration and end inspiration, respectively, and the average differences in $x$-, $y$-, and $z$-coordinates between inspiration and expiration were $0.56,2.20$, and $4.22 \mathrm{~mm}$, respectively.

compare this boundary condition with the one based on $\Delta v_{R}$, the correlation between the fraction of the cross-sectional area at TLC, $a_{\mathrm{TLC}}$, and $\Delta v_{\mathrm{R}}$ for ending branches was obtained (Figure 6(b)).

$\Delta v_{\mathrm{R}}=0.591( \pm 0.499) a_{\mathrm{TLC}} 0.842( \pm 0.176)(r=0.604( \pm 0.080))$

Applying the approximation $d V_{\mathrm{R}} / d V_{\mathrm{L}} \approx \Delta v_{\mathrm{R}} \approx a_{\mathrm{TLC}}$ to Equation 1 is equivalent to imposing a uniform velocity boundary condition at ending branches. Nonetheless, the poor correlation between $\Delta v_{\mathrm{R}}$ and $a_{\mathrm{TLC}}$ shown in Equation 12 indicates that a uniform velocity boundary condition is not appropriate for one-image-based models. 
Table 3. Average normalized absolute differences in airway cross-sectional area, airway length, volume, flow rate, pressure drop from the top of the trachea to the distal ends of 52 branches at peak inspiration compared to the case "default." The values are presented as \%, and the values in the parentheses are average normalized signed differences.

\begin{tabular}{crrrr}
\hline Case & 2 & 3 & 4 & 5 \\
Name & LD & RD & LS & RS \\
\hline Area & 1.6 & 5.4 & 5.5 & 5.1 \\
& $(+0.3)$ & $(+2.9)$ & $(-1.4)$ & $(+0.9)$ \\
Length & 1.5 & 6.2 & 5.3 & 5.7 \\
& $(-0.3)$ & $(+4.9)$ & $(-0.2)$ & $(+2.8)$ \\
Volume & 1.3 & 7.5 & 3.8 & 4.2 \\
& $( \pm 0.0)$ & $(+7.4)$ & $(-1.9)$ & $(+3.4)$ \\
Flow rate & - & - & 13.0 & 22.9 \\
& & & $(-0.3)$ & $(-0.5)$ \\
& & & & \\
Pressure drop & $(-1.9)$ & $(-13.4)$ & $(-1.9)$ & $(-5.8)$ \\
\hline
\end{tabular}

\subsection{Airway models based on dynamic and static images}

The effect of airway models constructed from dynamic vs. static scans was investigated via comparison of the LD and LS cases against the default case. The difference in pressure drop between the LS and default cases was 24.0 percentage points larger than that for the LD case (29.2\% vs. $5.2 \%$ in Table 3). The larger difference for the LS case can be attributable to the differences in airway geometry and flow-rate boundary condition (Figure 12). The difference in airway cross-sectional area and length between the LS and default cases was slightly larger than that between the LD and default case (about 5.4\% vs. 1.5\%). Moreover, the airway volume for 

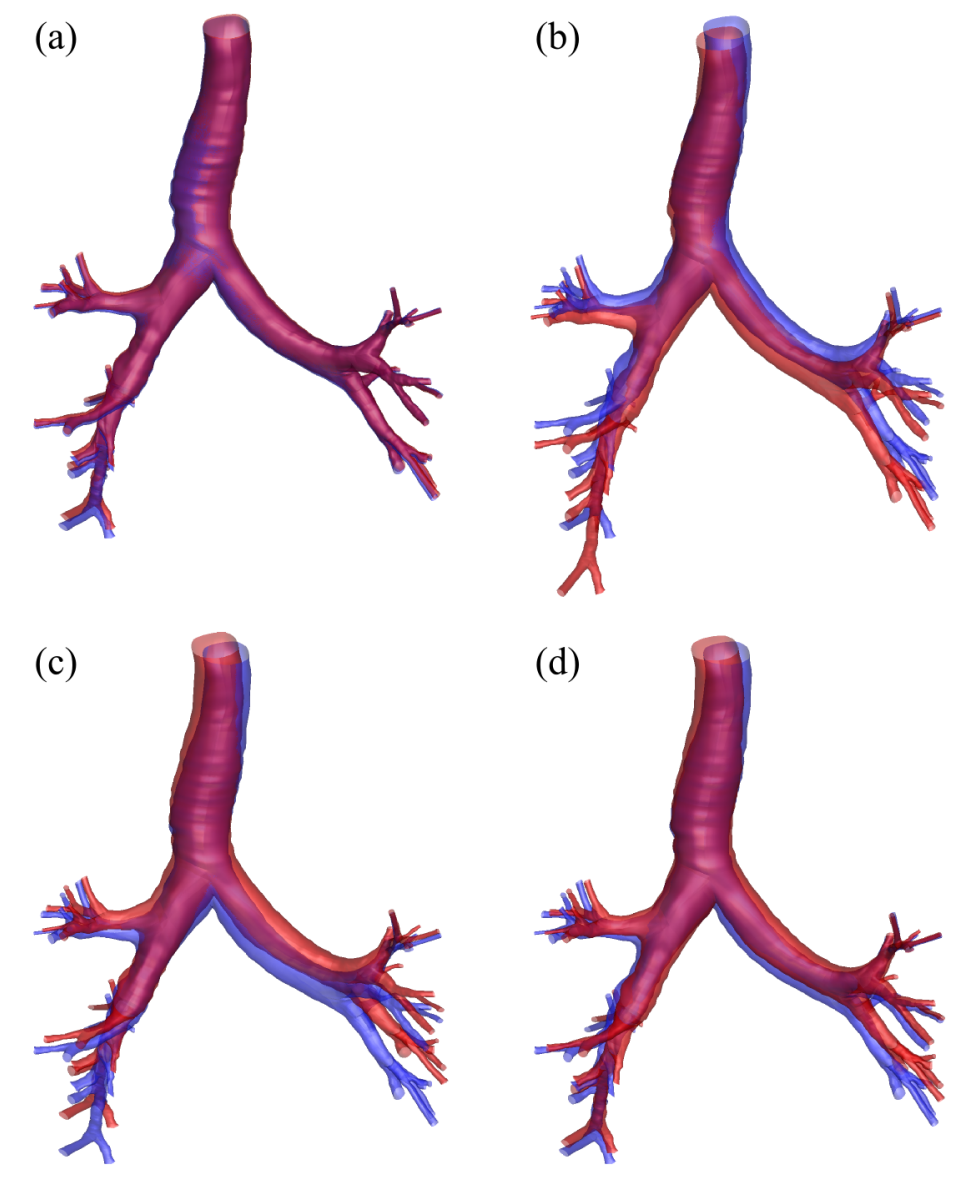

Figure 10. Surface meshes (red) of the (a) LD, (b) RD, (c) LS, and (d) RS cases compared to surface mesh of the default case (blue) at peak inspiration.

the LS case was slightly smaller than those of the default and LD cases $(-1.9 \%$ vs. $\pm 0.0 \%$ in Table 3). The smaller airway geometry for the LS case was also revealed by the z-coordinate of a surface node illustrated in Figure 3(b). As for the difference in flow rate between the LD and LS cases (13 percentage points), it was three times larger than that of geometry (the average of the differences in area and length) ( 3.9 percentage points).

As airflow structure determines the behavior of particles in the lung, we compared the 3D flow structures around the first bifurcation between the LD vs. LS cases (Figures 11(b)(c)). For 
the LS case, 9.5\% less air entered the RMB compared to the LD case, and the cross-sectional area at the distal end of the RMB was $10.0 \%$ larger for the LS case than the LD case. As a result, the bulk velocity was $18.7 \%$ slower in the RMB for the LS case, and the high-velocity region near the inner wall of the bifurcation that appeared in the LD case due to the inertia effect of bifurcating flow was nearly absent in the LS case. Furthermore, the difference in kinetic energy correction factor at the distal end of RMB was 9.2\%. In contrast, the difference in 3D flow structure at the distal end of the LMB between the LD and LS cases was not as clear as at the distal end of the RMB. The differences in bulk velocity and kinetic energy correction factor were 9.0\% and 3.2\%, respectively, at the distal end LMB.

\section{Discussion}

With advances in CT lung imaging technology, we progressively acquired two static images, three static images, and 4DCT images of the human lungs. The CFD lung models based on two and three static images were designed to investigate the effect of linear vs. non-linear deforming models on flow characteristics (Yin et al. (2013)). Nonetheless, 4DCT images can capture the hysteresis of lung deformation during tidal breathing (Jahani et al. (2015)), which is a key element in building a more physiologically realistic lung model and assessing the differences between static and dynamic imaging-based models. Thus, the objectives of this work were twofold. The first objective was to build a CFD model using 4DCT images. The other was to investigate the effects of relative hysteresis (a 4DCT-based model with relative hysteresis vs. a linearly deforming model in the absence of relative hysteresis), airway deformation (a linearly deforming model vs. a rigid model), and imaging method (models based on dynamic vs. static scans) on CFD results. 

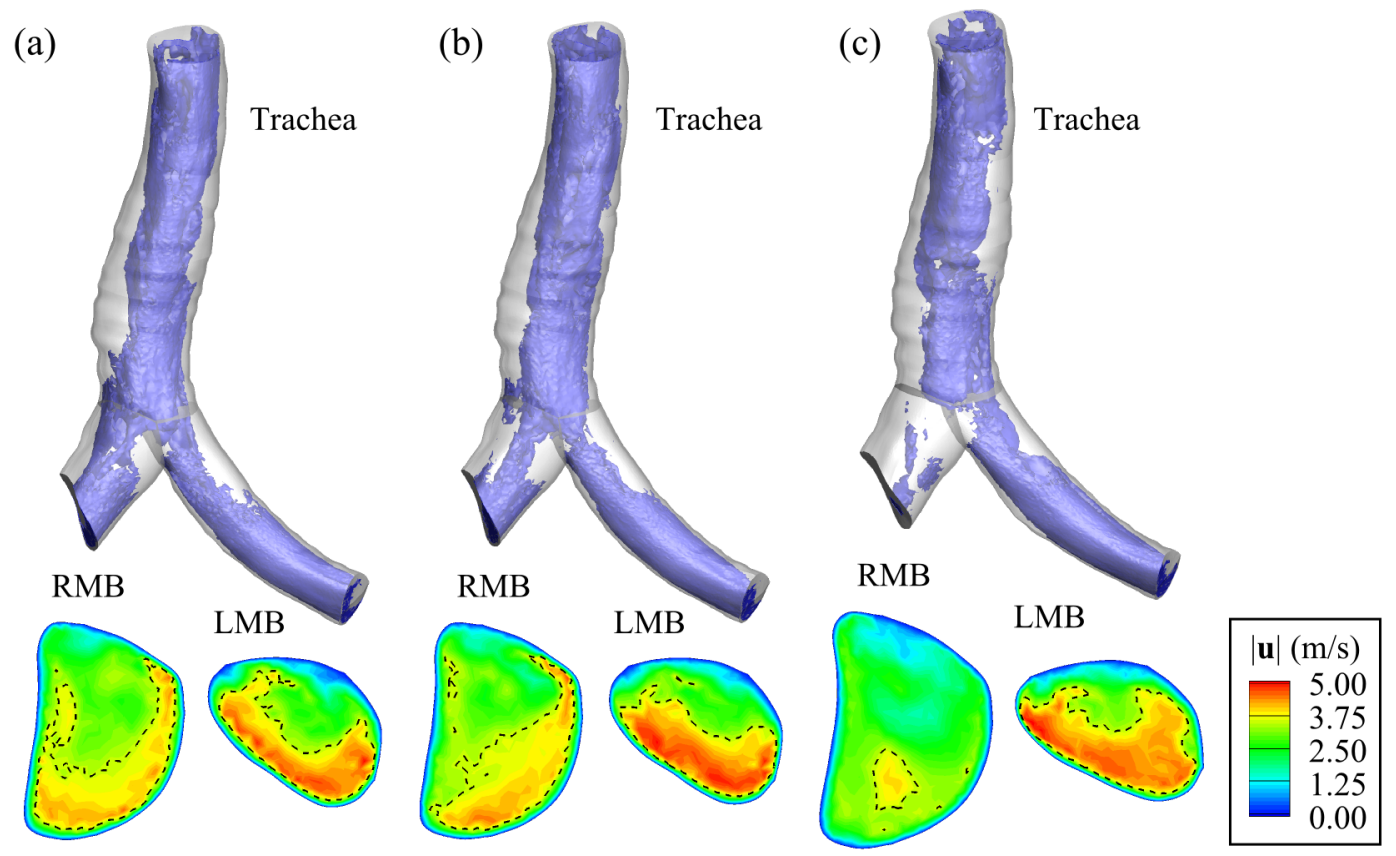

Figure 11. Instantaneous iso-surface of velocity magnitude $|\mathbf{u}|$ at $3.5 \mathrm{~m} / \mathrm{s}$ and velocity magnitude distribution at distal ends of left main bronchus (LMB) and right main bronchus (RMB) for (a) the default, (b) LD, and (c) LS cases at peak inspiration. The legend does not apply to the isosurface. The dash lines on the contour plots indicate $|\mathbf{u}|=3.5 \mathrm{~m} / \mathrm{s}$.

A 4DCT-based model was not merely a straightforward extension of our previous static image-based model (Yin et al. (2013)). The irregular airway motion derived from 4DCT data, consisting of volumetric lung images at 13 time points, required an improved moving meshing algorithm and the optimal interpolation method to construct smooth time-varying local variables without overshooting, which can cause numerical instability. Together with the static images of the same subject, we were able to quantitatively assess the CFD models and boundary conditions based on static and dynamic images.

For moving volume mesh, we adopted a CSM-based algorithm used by Stein et al. (2003) rather than a distance-function-based algorithm used by Yin et al. (2013). The former is 


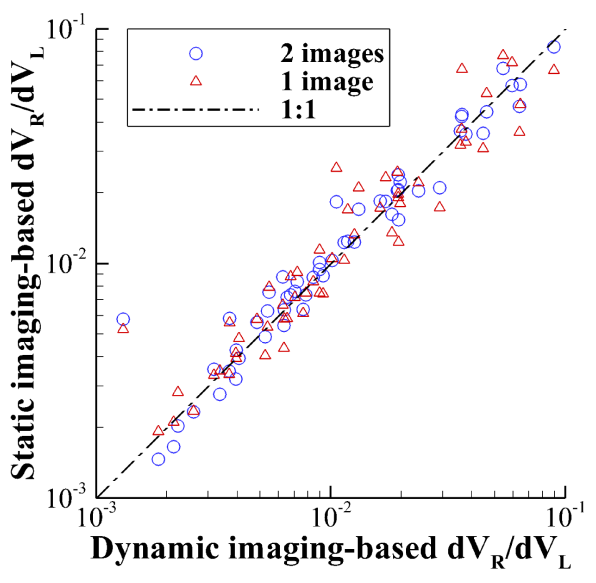

Figure 12. Flow rate fractions $\left(d V_{R} / d V_{L}\right)$ at distal ends of ending branches based on two dynamic images (default, LD, and RD case) vs. two static images (LS case) or one static image (RS case). The raw data are available in Supplementary Material.

computationally more expensive but more stable (by ensuring positive Jacobian of moving volume mesh) than the latter. The computational time for the CSM-based moving mesh solver was comparable to that of the fluid solver. The distance-function-based algorithm could cause negative Jacobian of volume mesh probably due to smaller mesh size and relatively large lung deformation between two consecutive time points in association with the uncertainty in 4DCT imaging (O’Connel et al. (2015)).

An important part of the proposed algorithm is concerned with the time rate of the change of a local variable $\phi(t)$, e.g., regional volume, which is expressed as $d \phi / d t=d \phi / d V_{L} \cdot d V_{L} / d t$, where $\phi(t)$ is decomposed into $\phi\left(V_{L}\right)$ and $V_{L}(t)$. This decomposition allows inspection of relative hysteresis for any imaging-based variable, e.g., $V_{R}\left(V_{L}\right)$ and $\mathbf{x}_{\mathrm{w}}\left(V_{L}\right)$ in Figures 2(b)(c), and employment of an arbitrary breathing waveform $V_{L}(t)$ given the same EI and EE lung volumes in the simulation. This feature distinguishes itself from the approach by Yin et al. (2013), which 
used a model based on three static images acquired at 78\%, 60\% and 20\% vital capacity (VC). Yin et al. (2013) assumed a sinusoidal waveform and the equivalence of $78 \%$ and $20 \%$ VC to the lung volumes at EI and EE, respectively, during tidal breathing. Then, the times corresponding to the three volumes were obtained to construct a cubic interpolation function for any local variable as a function of time.

With the new approach, the relationship between $V_{R}$ and $V_{L}$ was found to be nearly linear. This implies that the degree of hysteresis in $V_{R}$ is the same as that of $V_{L}$ with respect to the intrapleural pressure (Froeb and Mead (1968)). The linear $V_{R}\left(V_{L}\right)$ relationship greatly facilitated the imposition of flow boundary conditions at ending branches for CFD simulations. That is, with constant $d V_{R} / d V_{L}$ at ending branches, the distribution of airflow to ending branches remained unchanged regardless of time point. If a variable $\phi$ exhibits more hysteresis than lung volume, the relationship $\phi\left(V_{L}\right)$ has a counter-clockwise direction, and vice versa (Froeb and Mead (1968)). This is the case when $\phi=x$-, $y$-, or $z$-coordinate of the airway surface mesh as shown in Figures 8 and 9. A constrained cubic-spline interpolation function was then employed to approximate the non-linear relationship of $\mathbf{x}_{\mathrm{w}}\left(V_{L}\right)$ without overshooting. The $\mathbf{x}_{\mathrm{w}}\left(V_{L}\right)$ along with $V_{L}(t)$ enabled calculation of the displacement of the airway surface mesh between two consecutive time steps that is needed as an essential boundary condition to move volume mesh by solving Equation 7.

While a 4DCT-based lung model is most accurate in representing a breathing lung in vivo, it is not always possible to obtain 4DCT images due to increased radiation dose and other technical issues, e.g. controlling tidal breathing with consistent lung volumes over breathing cycles. Therefore, we compared a rigid model based on one static image (the RS case) and a linearly deforming model based on two static images (the LS case) against a non-linearly 
deforming model based on 4DCT images (the default case). For the rigid model based on one static image, we devised a new boundary condition, with which the flow rate at an ending branch is proportional to the fraction of regional volume of that branch. This was based on the observation that the fraction of change in regional volume associated with an ending branch $\left(\Delta \nu_{R}\right)$ was strongly correlated with the fraction of regional volume at TLC $\left(v_{\mathrm{R}, \mathrm{TLC}}\right)$ of the same branch (Figure 6(a) and Equation 4). Compared with the flow rates at ending branches in the default $4 \mathrm{DCT}$ case, the difference in the flow rate in the LS case was $13 \%$, whereas the difference based on the approximation $\Delta v_{\mathrm{R}} \approx v_{\mathrm{R}, \mathrm{TLC}}$ in the RS case was $22.9 \%$. Thus, the flow rate based on two static images is preferable if two static images are available. For some models based on one image, a uniform velocity boundary condition was applied to the ending branches. As a result, the flow rate at an ending branch depends on the cross-sectional area. A correlation analysis between the change in regional volume and the cross-sectional area indicated that a uniform velocity boundary condition is unlikely to produce physiologically realistic flow distribution (Equation 12).

Regarding the effect of dynamic vs. static imaging, i.e., the LD vs. LS cases, airway geometries constructed from dynamic and static images exhibited slight difference in dimension. Overall, the human airways were slightly larger during dynamic breathing than at static breathhold (Table 3 and Figure 3(b)), although the biases in pressure drop prediction quantified by average normalized signed difference were similar to each other as shown in Table 3. The dynamic vs. static imaging also affected the 3D flow structure, e.g., around the first bifurcation (Figures 11(b)(c)). Due to the difference in geometry and flow rate in RMB, velocity near the inner wall was not amplified for the LS case as much as for the LD case. This could cause a difference in the distribution of particles passing through the RMB between the two cases. 
Particles would not be mixed well in radial direction for the LS case compared to the LD case because of the weaker secondary flow in the former.

We also evaluated the effects of relative hysteresis (the 4DCT default case vs. the linearly deforming LD case) and airway deformation (the linearly deforming LD case vs. the rigid RD case). An inspection of the relative hysteresis of the airway surface mesh $\mathbf{x}_{\mathrm{w}}\left(V_{L}\right)$ in the default case showed that more hysteresis existed along the dorsal-ventral $(y)$ axis and the cephaladcaudad ( $z)$ axis than along the left-right $(x)$ axis. Since the bulk difference in geometry between the default and LD cases was only on the order of $1.5 \%$, the bulk difference of $5.2 \%$ in pressure drop between them was not large. Nonetheless, the LD case may not be able to capture sensitively time-accurate pressure variation during tidal breathing. In comparison of LD vs. RD cases, a greater difference in geometry was found in the RD case, leading to under-predicted pressure drop.

One of the limitations of this study is that we considered one healthy subject, so for the future work, we may apply the model to asthmatic 4DCT data and compare flow characteristics and lung deformation in healthy and asthmatic subjects. For instance, Choi et al. (2013) demonstrated that severe asthmatics are characterized by significant alterations of regional airvolume distribution and lung deformation. This study implies that asthma may cause significantly different flow characteristics associated with pressure drops observed when one evaluates the dynamic images rather than static conditions. In addition, as one of the objectives of this study was to develop a CFD model for a deforming human lung using 4DCT images, we did not investigate the flow characteristics in great detail, which could be addressed in the future work together with particle distribution. To allow other researchers to further interrogate the 
current data set, airway geometries and CFD flow field data can be obtained by contacting the corresponding author.

In summary, we developed a CFD lung model based on 4DCT images and compared it with other simplified models based on one or two dynamic and static images. The difference between dynamic vs. static imaging significantly affected pressure drop along central airways due to the differences in flow distribution and airway geometry. In a similar way, prediction of particle delivery to the human lung could be also affected by dynamic vs. static imaging. The human lung models presented in this paper would enable researchers to select their optimal model, depending on the availability of data and computational resources.

\section{Acknowledgements}

This work was supported in part by NIH grants R01-HL094315, U01-HL114494, R01HL112986, S10-RR024738, and S10-RR022421. We would like to thank Nariman Jahani for his help with utilization of the 4D CT data and associated physiologic parameters. We also thank the Extreme Science and engineering Discovery Environment (XSEDE) (allocation MCA07S015) sponsored by the National Science Foundation that provided the computational time at San

Diego Supercomputer Center (SDSC) and the Texas Advanced Computing Center (TACC).

\section{References}

1. Bhatt, S. P., S. Bodduluri, J. D. Newell, E. A. Hoffman, J. C. Sieren, M. K. Han, M. T. 
Dransfield, and J. M. Reinhardt. CT-derived Biomechanical Metrics Improve Agreement Between Spirometry and Emphysema. Acad. Radiol. (in press).

2. Bodduluri, S., J. D. Newell Jr, E. A. Hoffman, and J. M. Reinhardt. Registration-Based Lung Mechanical Analysis of Chronic Obstructive Pulmonary Disease (COPD) Using a Supervised Machine Learning Framework. Acad. Radiol. 20:527-536, 2013.

3. Choi, J., M. H. Tawhai, E. A. Hoffman, and C. L. Lin. On intra-and intersubject variabilities of airflow in the human lungs. Phys. Fluids 21:101901, 2009.

4. Choi, S., E. A. Hoffman, S. E. Wenzel, M. H. Tawhai, Y. Yin, M. Castro, and C.-L. Lin. Registration-based Assessment of Regional Lung Function via Volumetric CT Images of Normals vs. Severe Asthmatics. J. Appl. Physiol., 2013.

5. Froeb, H. F., and J. Mead. Relative hysteresis of the dead space and lung in vivo.. J. Appl. Physiol. 25:244-248, 1968.

6. Fuld, M. K., R. W. Grout, J. Guo, J. H. Morgan, and E. A. Hoffman. Systems for Lung Volume Standardization during Static and Dynamic MDCT-based Quantitative Assessment of Pulmonary Structure and Function. Acad. Radiol. 19:930-940, 2012.

7. Galbán, C. J., M. K. Han, J. L. Boes, K. A. Chughtai, C. R. Meyer, T. D. Johnson, S. Galbán, A. Rehemtulla, E. A. Kazerooni, F. J. Martinez, and others. Computed tomography-based biomarker provides unique signature for diagnosis of COPD phenotypes and disease progression. Nat. Med. 18:1711-1715, 2012.

8. Hoffman, E. A., B. A. Simon, and G. McLennan. State of the Art. A Structural and Functional Assessment of the Lung via Multidetector-Row Computed Tomography. Proc. Am. Thorac. Soc. 3:519--532, 2006.

9. Jahani, N., S. Choi, J. Choi, K. Iyer, E. Hoffman, and C.-L. Lin. Assessment of Regional Ventilation and Deformation Using 4D-CT Imaging for Healthy Human Lungs during 
Tidal Breathing. J. Appl. Physiol. Accepted:, 2015.

10. Jahani, N., Y. Yin, E. A. Hoffman, and C.-L. Lin. Assessment of regional non-linear tissue deformation and air volume change of human lungs via image registration. J. Biomech. 47:1626--1633, 2014.

11. Jarrin, N., S. Benhamadouche, D. Laurence, and R. Prosser. A synthetic-eddy-method for generating inflow conditions for large-eddy simulations. Int. J. Heat Fluid Flow 27:585593, 2006.

12. Kruger, C.. Constrained cubic spline interpolation. Chemical Engineering Applications, 2003.

13. Lin, C. L., M. H. Tawhai, G. McLennan, and E. A. Hoffman. Characteristics of the turbulent laryngeal jet and its effect on airflow in the human intra-thoracic airways. Respiratory physiology \& neurobiology 157:295-309, 2007.

14. Loring, S. H., C. R. O'Donnell, D. J. Feller-Kopman, and A. Ernst. Central airway mechanics and flow limitation in acquired tracheobronchomalacia*. Chest 131:1118-1124, 2007.

15. Mead, J., J. L. Whittenberger, and E. P. Radford. Surface Tension as a Factor in Pulmonary Volume-Pressure Hysteresis. J. Appl. Physiol. 10:191--196, 1957.

16. Mead-Hunter, R., A. J. King, A. N. Larcombe, and B. J. Mullins. The influence of moving walls on respiratory aerosol deposition modelling. J. Aerosol Sci. 64:48-59, 2013.

17. Miyawaki, S., M. H. Tawhai, E. A. Hoffman, and C.-L. Lin. Effect of carrier gas properties on aerosol distribution in a CT-based human airway numerical model. Ann. Biomed. Eng. 40:1495-1507, 2012.

18. Miyawaki, S., M. H. Tawhai, E. A. Hoffman, and C.-L. Lin. An automatic generation of non-uniform mesh for CFD analyses of image-based multiscale human airway models. 
Bulletin of the American Physical Society 59:, 2014.

19. Miyawaki, S., M. H. Tawhai, E. A. Hoffman, and C.-L. Lin. An image-based automatic mesh generation and numerical simulation for a population-based analysis of aerosol delivery in the human lungs. Bulletin of the American Physical Society, 2013.

20. O'Connell, D. P., D. H. Thomas, T. H. Dou, J. M. Lamb, F. Feingold, D. A. Low, M. K. Fuld, J. P. Sieren, C. M. Sloan, M. A. Shirk, E. A. Hoffman, and C. Hofmann. Comparison of breathing gated CT images generated using a 5DCT technique and a commercial clinical protocol in a porcine model. Med. Phys. 42:4033-4042, 2015.

21. Press, W. H., S. A. Teukolsky, T. Veterling, and B. Flannery. Numerical recipes 3rd edition. The art of scientific computing, 2007.

22. Ramsey, J. B.. Tests for Specification Errors in Classical Linear Least-Squares Regression Analysis. Journal of the Royal Statistical Society. Series B (Methodological) 31:350--371, 1969.

23. Sera, T., H. Yokota, R. Himeno, and G. Tanaka. Gas dispersion of oscillatory flow in expanding and contracting multi-branching airways. Int. J. Heat Mass Transfer 65:627634, 2013.

24. Stein, K., T. Tezduyar, and R. Benney. Mesh moving techniques for fluid-structure interactions with large displacements. Journal of Applied Mechanics 70:58, 2003.

25. Tawhai, M. H., A. Pullan, and P. Hunter. Generation of an anatomically based threedimensional model of the conducting airways. Ann. Biomed. Eng. 28:793-802, 2000.

26. Vreman, A. W.. An eddy-viscosity subgrid-scale model for turbulent shear flow: Algebraic theory and applications. Phys. Fluids 16:3670, 2004.

27. Wall, W. A., and T. Rabczuk. Fluid-structure interaction in lower airways of CT-based lung geometries. Int. J. Numer. Methods Fluids 57:653-675, 2008. 
28. Wu, D., M. Tawhai, E. Hoffman, and C.-L. Lin. A Numerical Study of Heat and Water Vapor Transfer in MDCT-Based Human Airway Models. Ann. Biomed. Eng. 42:21172131, 2014.

29. Xia, G., M. H. Tawhai, E. A. Hoffman, and C. L. Lin. Airway Wall Stiffening Increases Peak Wall Shear Stress: A Fluid-Structure Interaction Study in Rigid and Compliant Airways. Ann. Biomed. Eng. 38:1836-1853, 2010.

30. Yin, Y., J. Choi, E. A. Hoffman, M. H. Tawhai, and C.-L. Lin. A multiscale MDCT imagebased breathing lung model with time-varying regional ventilation. J. Comput. Phys. 244:168-192, 2013.

31. Yin, Y., E. A. Hoffman, K. Ding, J. M. Reinhardt, and C. L. Lin. A cubic B-spline-based hybrid registration of lung CT images for a dynamic airway geometric model with large deformation. Phys. Med. Biol. 56:203, 2011. 